\title{
Vascular endothelial growth factor $A$, secreted in response to transforming growth factor- $\beta 1$ under hypoxic conditions, induces autocrine effects on migration of prostate cancer cells
}

\author{
Eric Darrington*, Miao Zhong*, Bao-Han Vo and Shafiq A Khan*
}

Hypoxia and transforming growth factor- $\beta 1$ (TGF- $\beta 1$ ) increase vascular endothelial growth factor $A$ (VEGFA) expression in a number of malignancies. This effect of hypoxia and TGF- $\beta 1$ might be responsible for tumor progression and metastasis of advanced prostate cancer. In the present study, TGF- $\beta 1$ was shown to induce VEGFA 165 secretion from both normal cell lines (HPV7 and RWPE1) and prostate cancer cell lines (DU145 and PC3). Conversely, hypoxia-stimulated VEGFA 165 secretion was observed only in prostate cancer cell lines. Hypoxia induced TGF- $\beta 1$ expression in PC3 prostate cancer cells, and the TGF- $\beta$ type I receptor (ALK5) kinase inhibitor partially blocked hypoxia-mediated VEGFA 165 secretion. This effect of hypoxia provides a novel mechanism to increase VEGFA expression in prostate cancer cells. Although autocrine signaling of VEGFA has been implicated in prostate cancer progression and metastasis, the associated mechanism is poorly characterized. VEGFA activity is mediated via VEGF receptor (VEGFR) 1 (FIt-1) and 2 (FIk-1/KDR). Whereas VEGFR-1 mRNA was detected in normal prostate epithelial cells, VEGFR-2 mRNA and VEGFR protein were expressed only in PC3 cells. VEGFA 165 treatment induced phosphorylation of extracellular signal-regulated kinase $1 / 2$ (ERK1/2) in PC 3 cells but not in HPV7 cells, suggesting that the autocrine function of VEGFA may be uniquely associated with prostate cancer. Activation of VEGFR-2 by VEGFA 165 was shown to enhance migration of PC 3 cells. A similar effect was also observed with endogenous VEGFA induced by TGF- $\beta 1$ and hypoxia. These findings illustrate that an autocrine loop of VEGFA via VEGFR-2 is critical for the tumorigenic effects of TGF- $\beta 1$ and hypoxia on metastatic prostate cancers.

Asian Journal of Andrology (2012) 14, 745-751; doi:10.1038/aja.2011.197; published online 18 June 2012

Keywords: cell migration; hypoxia; prostate cancer; transforming growth factor- $\beta 1$ (TGF- $\beta 1$ ); vascular endothelial growth factor $A$ (VEGFA)

\section{INTRODUCTION}

Vascular endothelial growth factor A (VEGFA) belongs to a family of angiogenic growth factors. ${ }^{1}$ To date, at least four VEGFA isoforms $\left(\right.$ VEGFA $\left._{121}, 165,189,206\right)$ have been identified. ${ }^{1}$ VEGFA $_{165}$ is considered the predominant VEGFA isoform because it mimics the full spectrum of VEGFA functions. ${ }^{1,2}$ The bioactivities of VEGFA are mediated by two different types of receptor tyrosine kinases, VEGF receptor 1 (VEGFR-1, Flt-1) and VEGFR-2 (Flk-1/KDR). ${ }^{3,4}$ Neuropilin-1 is another membrane protein that does not have any intrinsic kinase activity but binds to VEGFA $_{165}$ with high affinity. ${ }^{2}$ VEGFA has been shown to be overexpressed in solid tumors of different origins. ${ }^{1}$ In addition to the paracrine role in endothelial cells to induce tumor neovascularization, autocrine effects of VEGFA in tumor progression and metastasis have recently been reported in a number of malignancies. ${ }^{5}$

Hypoxia is a general event that occurs concomitant with tumor growth. ${ }^{1}$ Hypoxia stabilizes the hypoxia-inducible factor-1 (HIF-1) complex, which causes an increase in VEGFA gene expression. ${ }^{6}$ The HIF-binding element has been identified in the promoter region of the human VEGFA gene, along with the Smad-binding elements in the proximal region. ${ }^{7,8}$ Transforming growth factor- $\beta$ (TGF- $\beta$ ) signaling plays an important role in tumor angiogenesis. ${ }^{9}$ TGF- $\beta 1$ signaling has been shown in concert with HIF- $1 \alpha$ to regulate VEGFA expression. ${ }^{7,8}$ Hypoxia also increases TGF- $\beta 1$ expression in osteoblast and hepatoma cells. ${ }^{10,11}$ Hence, TGF- $\beta 1$ signaling may constitute a positive feedback loop to reinforce the effect of hypoxia on VEGFA expression.

A consistent increase in VEGFA expression has been observed in primary tumor specimens as well as serum samples from prostate cancer patients. ${ }^{12,13}$ Anti-VEGFA treatment has been proven to be effective anti-cancer therapy to prevent prostate cancer progression. ${ }^{14}$ Whereas the paracrine role of VEGFA to induce tumor neovascularization has been extensively characterized, very little is known about its autocrine effects on prostate cancer growth and metastasis. A functional VEGFR-1 has been identified in a tumorigenic derivative of rat 
prostate epithelial cell line. ${ }^{15}$ Currently, data on VEGFR-2 expression in prostate cancer cells are still controversial. ${ }^{16,17}$

In the present study, we examined the effects of TGF- $\beta 1$ on VEGFA secretion under normal and hypoxic conditions in normal and prostate cancer cell lines. We also examined the effect of VEGFA 165 on migration and proliferation of PC3 cells. The potential influence of hypoxia on TGF- $\beta 1$ expression and the resulting autocrine effect on VEGFA 165 secretion were also investigated in PC3 cells. Our data support that VEGFA is a critical autocrine regulator for the tumorigenic effects of hypoxia and TGF- $\beta 1$ in metastatic prostate cancer cells.

\section{MATERIALS AND METHODS}

\section{Reagents}

Recombinant human VEGFA 165 was obtained from Peprotech (Rocky Hill, NJ, USA). Soluble VEGFR-2 was obtained from Prospec (East Brunswick, NJ, USA). Ki8751 and SB431542 were obtained from Tocris (Park Elisville, MO, USA). QuantiGlo human VEGF immunoassay kit, Quantikine human TGF- $\beta 1$ immunoassay kit, and recombinant human TGF- $\beta 1$ were obtained from R\&D Systems (Minneapolis, MN, USA). All primers were purchased from IDT (San Jose, CA, USA). Dc protein assay kit was obtained from Bio-Rad (Hercules, CA, USA). Cell culture reagents were obtained from Mediatech Inc. (Manassas, VA, USA).

\section{Cell culture and cell treatments}

Immortalized prostate luminal epithelial cell lines (RWPE1 and HPV7), and prostate cancer cell lines (DU145 and PC3) were obtained from American Type Culture Collection (ATCC, Rockville, MD, USA). RWPE1 and HPV7 cell lines were maintained in Keratinocyte growth medium supplemented with $0.05 \mathrm{mg} \mathrm{ml}^{-1}$ bovine pituitary extracts and $5 \mathrm{ng} \mathrm{ml}^{-1}$ epidermal growth factor (EGF; Invitrogen, Carlsbad, CA, USA). DU145 and PC3 cell lines were maintained in Eagle's minimum essential medium supplemented with $5 \%$ fetal bovine serum.

Cells were seeded at a density of $1.5 \times 10^{5}$ per well in six-well culture plates for 2 days. The next day, cells were treated as described in the figure legends in culture medium containing $0.2 \%$ bovine serum albumin (Sigma, St Louis, MO, USA). Hypoxia was achieved with a BillupsRothlesburg chamber (ACME manufacturing, Inc., Springfield, OR, USA) filled with premixed $94 \% \mathrm{~N}_{2}, 5 \% \mathrm{CO}_{2}$ and $1 \% \mathrm{O}_{2}$.

\section{Enzyme-linked immunoassay (ELISA)}

After treatments, conditioned media (CM) and cell lysates from RWPE1, HPV7, DU145 and PC3 cell lines were harvested. ${ }^{18}$
Concentrations of VEGFA 165 protein were measured with an ELISA kit according to the instruction provided by the manufacturer (R\&D Systems). Media incubated without cells and the cell lysis buffer were used as controls. Protein concentrations in cell lysates were measured as described previously. ${ }^{18}$

CM and cell lysates were collected from PC3 cells treated with or without hypoxia. Levels of TGF- $\beta 1$ were measured with an ELISA kit according to the instruction provided by the manufacturer.

\section{Western blot}

Total cell membranes from HPV7, DU145 and PC3 cell lines were prepared as described previously. ${ }^{19}$ Total cell proteins $(30 \mu \mathrm{g})$ of human umbilical vein endothelial cells were used as a positive control for VEGFR-2. The membrane proteins $(60 \mu \mathrm{g})$ were separated on $7 \%$ sodium dodecyl sulfate-polyacrylamide gel electrophoresis (SDS-PAGE) gels and transferred onto polyvinylidene fluoride membranes. VEGFR-2 was detected with anti-VEGFR-2 antibody ( 1 : 1000; Cell Signaling, Danvers, MA, USA). Anti- $\alpha$-tubulin antibody ( $1: 1000$; Sigma) was used to detect $\alpha$-tubulin as a sample loading control. To examine phosphorylation of extracellular signal-regulated kinase $1 / 2($ ERK1/2), total cell proteins $(30 \mu \mathrm{g})$ were separated on $10 \%$ SDS-PAGE gels. Anti-phospho-ERK1/2 antibody (1:2000; Cell Signaling) and anti-ERK1/2 antibody (1:5000; Promega, Madison, WI, USA) were used. Anti-phospho-Smad3 antibody (1:1000; Cell Signaling) and anti-Smad2/3 antibody ( $1: 1000$; Cell Signaling) were used to detect TGF- $\beta$ signaling in PC3 samples treated with or without hypoxia. The signal was detected by ECL (Thermo Scientific, Rockford, IL, USA).

\section{RNA extraction, cDNA synthesis, PCR and quantitative real-time PCR}

The primers for TGF- $\beta 1, V E G F A, V E G F R-1, V E R F R-2$ and L19 were designed as described previously ${ }^{18}$ and listed in Table 1 . Total RNAs from RWPE1, HPV7, DU145 and PC3 cell lines were reverse transcribed as described previously. ${ }^{18}$ PCR was performed as previously described. ${ }^{18}$ Real-time PCR was performed in the Bio-Rad icycler thermal cycler. ${ }^{18}$ Calculations were performed using the $\Delta \Delta C_{\mathrm{t}}$ method. ${ }^{18}$

\section{Cell migration assay}

Conditioned media and diluted VEGFA 165 were used to examine the effects of endogenous and exogenous VEGFA on in vitro migration of PC3 cells. The assay was performed using 24 -well transwell inserts $(8 \mu \mathrm{m}$; BD Biosciences:San Jose, CA, USA) as described previously. ${ }^{18}$ Data were expressed as migration index defined as: the average number of cells per

Table 1 Primers for RT-PCR and quantitative PCR of the indicated genes

\begin{tabular}{lllll}
\hline Genes & Gene ID & Primers & Sequences (5'-3') & Location \\
\hline TGF- $\beta 1$ & 260655621 & TGF 1 Product size $($ bp) & TCGATTTTGACGTCACTGGAGTTGT & $1473-93$ \\
& & TGF $\beta 1 R$ & GGGGTGGCCATGAGGAGCAGG & $1673-53$ \\
VEGFA & 284172452 & VEGFA-F & CAAGACAAGAAATCCCTGTGG & $1448-69$ \\
& & VEGFA-R & GCTTGTCACATCTGCAAGTACG & $1603-1582$ \\
VEGFR-1 & 229892268 & VEGFR1F & CCTTGAACACAGCTCAAGCA & $563-582$ \\
& & VEGFR1R & CCCAGATTATGCGTTTCCA & $844-825$ \\
VEGFR-2 & 2655411 & VEGFR2F & GCTTTGGCCCAATAATCAGA & $486-505$ \\
& & VEGFR2R & ACACGACTCCATGTTGGTCA & $712-693$ \\
L19 & L19-F & GAAATCGCCAATGCCAACTC & $306-325$ \\
& & L19-R & TCTTAGACCTGCGAGCCTCA & $711-692$ \\
\hline
\end{tabular}

Abbreviations: RT-PCR, reverse transcription PCR; TGF- $\beta 1$, transforming growth factor- $\beta 1$; VEGFA, vascular endothelial growth factor A; VEGFR, vascular endothelial growth factor receptor. 
field for test substance divided by the average number of cells per field for the medium control.

\section{Statistical analysis}

Data from multiple independent experiments are expressed as mean \pm SEM. Paired $t$-test and ANOVA and Duncan's modified multiple range test were used to examine significance between different treatments. $P<0.05$ was considered statistically significant.

\section{RESULTS}

VEGFA $_{165}$ is differentially regulated by TGF- $\beta 1$ and hypoxia in normal and prostate cancer cells

Quantitative real-time PCR was used to determine basal levels of VEGFA mRNA in normal prostate epithelial cell lines (RWPE1 and HPV7), as well as in DU145 and PC3 prostate cancer cell lines. VEGFA mRNA levels were comparable between the normal prostate epithelial cell lines (data not shown). However, VEGFA mRNA levels were twofold higher in PC3 and 25-fold higher in DU145 cell lines, when compared to RWPE1 cells (data not shown).

VEGFA $_{165}$ is the predominant active VEGFA isoform. Conditioned media and cell lysates were harvested from the four cell lines; the concentrations of VEGFA 165 were quantified with ELISA. The levels of VEGFA ${ }_{165}$ were $6.13,4.81,7.33$ and $2.43 \mathrm{pg} \mathrm{mg}^{-1}$ protein in the cell lysates from RWPE1, HPV7, DU145 and PC3 cells, respectively (Figure 1a). The conditioned media contained the majority of VEGFA $_{165}$ protein. As shown in Figure 1b, DU145 secreted at least 10 times higher levels of VEGFA VI65 $_{1}$ than RWPE1 and HPV7 cells (4493, 153 and $412 \mathrm{pg} \mathrm{mg}^{-1}$ protein for DU145, RWPE1 and HPV7 cells, respectively). PC3 cells secreted the least amount of VEGFA 165 (19 $\mathrm{pg} \mathrm{mg}^{-1}$ protein).

The four cell lines were treated with $10 \mathrm{ng} \mathrm{ml}^{-1}$ of TGF- $\beta 1$ under normal and hypoxic conditions. As shown in Figure 1c, TGF- $\beta 1$ stimulated VEGFA 165 secretion in both RWPE1 (threefold over control) and HPV7 (2.4-fold over control) cell lines; no additive effect of TGF$\beta 1$ was observed under hypoxia in either cell line. However, TGF- $\beta 1$ induced less than twofold increases in VEGFA VI5 $_{16}$ secretion in DU145 and PC 3 cell lines, which were enhanced to more than threefold under hypoxic conditions.
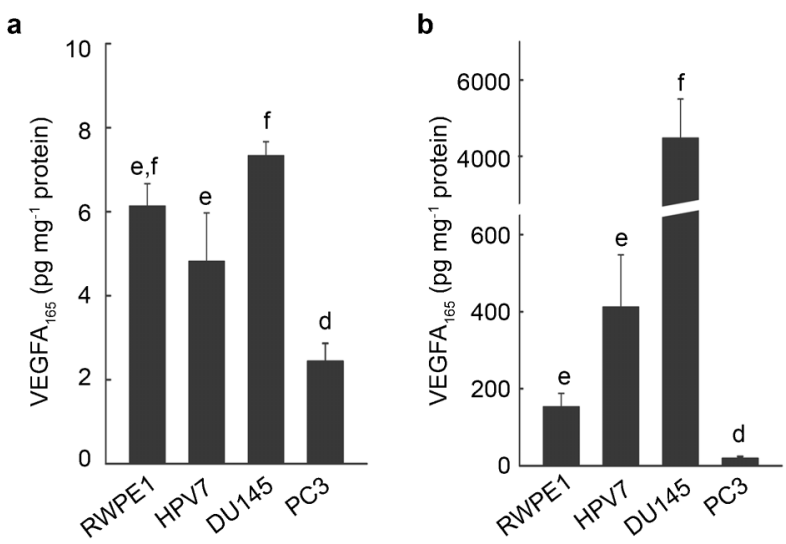

TGF- $\beta 1$ is an autocrine regulator of hypoxia-mediated VEGFA $A_{165}$ secretion in prostate cancer cells.

TGF- $\beta 1$ mRNA and TGF- $\beta 1$ protein levels were increased in PC 3 cells after exposure to hypoxia (Figure $2 \mathbf{a}$ and $\mathbf{b}$ ). Hypoxia induced the phosphorylation of Smad3 in PC3 cells (Figure 2c), consistent with the involvement of TGF- $\beta 1$ signaling in the effects of hypoxia. TGF- $\beta 1$ has been shown to induce VEGFA secretion via a Smad3-dependent mechanism. ${ }^{20}$ Pretreatment with SB431542, a TGF- $\beta$ receptor I (TGFßR-I, ALK5) inhibitor, ${ }^{21}$ reduced basal VEGFA ${ }_{165}$ secretion in PC3 cells (Figure 2d). TGF- $\beta 1$ ( $\left.1 \mathrm{ng} \mathrm{ml}^{-1}\right)$ induced a 1.4-fold increase in VEGFA 165 secretion, which was completely blocked by SB431542 (Figure 2d). Hypoxia induced a more than twofold increase in VEGFA $_{165}$ secretion, which was partially blocked by SB431542 (Figure 2d). Hypoxia plus TGF- $\beta 1$ treatment induced a fourfold increase in $\mathrm{VEGFA}_{165}$ secretion; however, the combined effect of hypoxia and TGF- $\beta 1$ was reduced by SB431542 to a level that is similar to the treatment of hypoxia plus SB431542 (Figure 2d).

\section{VEGFR-2 is involved in VEGFA functions in PC3 cells}

VEGFR- 1 and VEGFR-2 mRNA were determined by reverse transcription (RT)-PCR in RWPE1, HPV7, DU145 and PC3 cell lines. As shown in Figure 3a, VEGFR-1 mRNA was detected in the normal prostate cell lines. VEGFR-2 was expressed only in the prostate cancer cell lines. Total cell membranes were prepared from HPV7, DU145 and PC 3 cell lines, and were analyzed for VEGFR-2 expression by western blot. Total cell lysate from human umbilical vein endothelial cells (HUVEC) was used as a positive control. Western blot for $\alpha$-tubulin were performed for sample-loading control. As shown in Figure $\mathbf{3 b}$, VEGFR-2 protein was not detectable in HPV7 cells, consistent with the absence of VEGFR-2 mRNA in this cell line. Although VEGFR-2 mRNA was expressed in both DU145 and PC3 cell lines, VEGFR-2 protein was detected only in PC3 cells (Figure $3 \mathbf{b}$ ). TGF- $\beta 1$ (1 ng $\mathrm{ml}^{-1}$ ) induced ERK1/2 phosphorylation in both HPV7 and PC3 cell lines. VEGFA 165 induced ERK1/2 phosphorylation only in PC3 cells (Figure 3c). Activated VEGFR-1 has been shown to induce ERK1/2 phosphorylation in epidermal tumors. ${ }^{5}$ Hence, VEGFA signaling is absent in HPV7 cells, despite the fact that VEGFR-1 mRNA is expressed in this cell line.

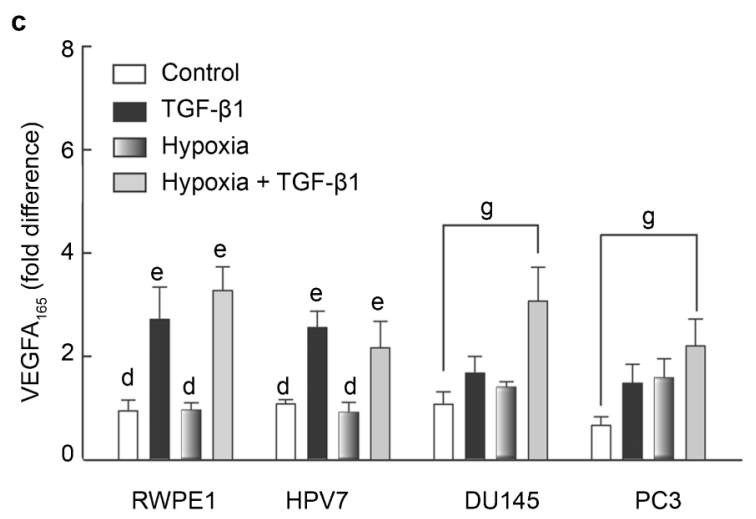

Figure 1 Differential regulation of VEGFA 165 secretion by TGF- $\beta 1$ and hypoxia in human prostate cell lines. Normal cell line (RWPE1 and HPV7) and prostate cancer cell lines (DU145 and PC3) were treated with or without TGF- $\beta 1$ (10 ng ml ${ }^{-1}$ ) in normoxic and hypoxic conditions for 7 h. Concentrations of VEGFA 165 in cell lysates and CM were quantified by ELISA. (a) Basal levels of VEGF165 in cell lysates from RWPE1, HPV7, DU145, and PC3 cell lines. (b) Basal levels of VEGF165 in conditioned media from RWPE1, HPV7, DU145, and PC3 cells lines, and (c) fold changes over basal levels of secreted VEGFA 165 for TGF- $\beta 1$ - and hypoxia-induced VEGFA 165 secretion in the four cell lines. Data are expressed as mean \pm SEM $(n=3)$, and were analyzed by ANOVA and Duncan's modified range tests. Significant differences between groups in a given category $(P<0.05)$ are designated with different lowercase letters. $P<0.05$ for $t$-test. CM, conditioned media; ELISA, enzyme-linked immunoassay; TGF- $\beta 1$, transforming growth factor- $\beta 1$; VEGFA, vascular endothelial growth factor $A$. 
a

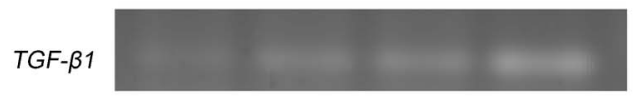

L19

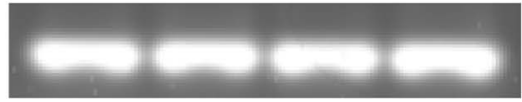

Hypoxia (h)

Folds:

0

0.5

1

2

5.94

6.03

7.88

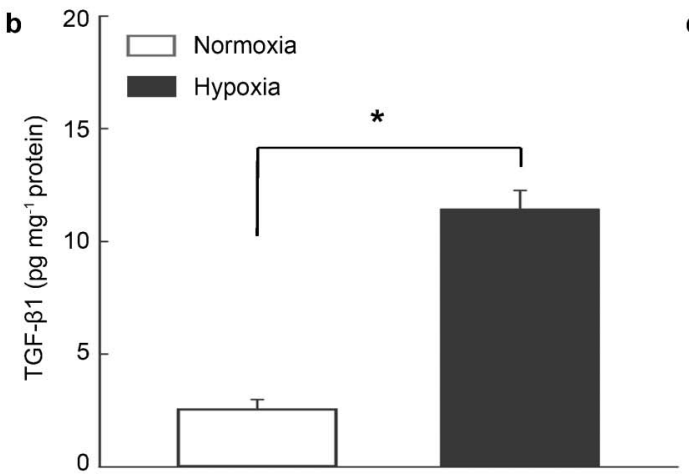

c

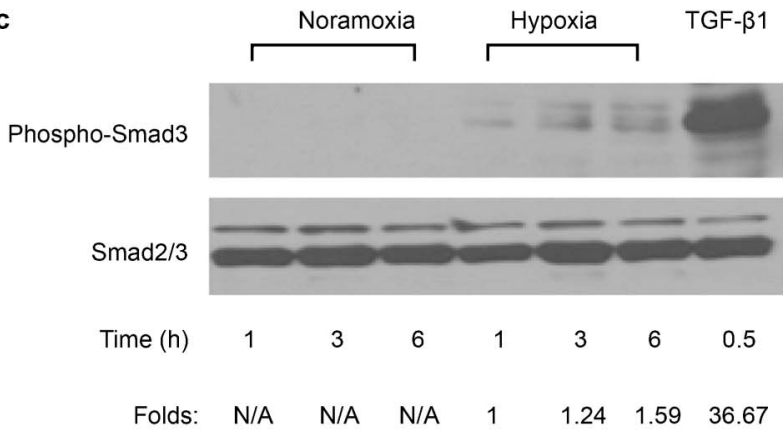

d

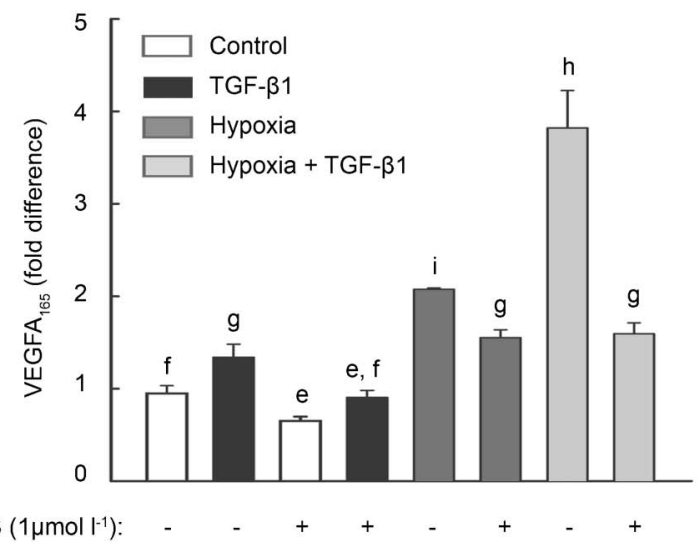

Figure 2 Autocrine action of TGF- $\beta 1$ on hypoxia-induced VEGFA $A_{165}$ secretion in PC3 cells. (a) PC3 cells were exposed to hypoxia for different times. TGF- $\beta 1$ mRNA levels were detected by RT-PCR. L19 gene expression was used as a control. Density of TGF- $\beta 1$ and L19 bands were quantified with ImageQuantTL software (GE Healthcare). Fold changes over the control group were calculated. Similar results were replicated in three independent experiments. (b) PC3 cells were treated with or without hypoxia for $6 \mathrm{~h}$. Concentrations of TGF- $\beta 1$ were quantitated with an immunoassay kit in samples of cell lysates, and were normalized with total protein concentrations $(* P<0.001$ ). (c) After hypoxic treatments, PC3 cells were collected in cell lysis buffer. Samples (35 $\mu$ g protein per lane) were separated on $10 \%$ SDSPAGE and then transferred on PVDF membrane. Phosphorylation of Smad3 was analyzed with anti-phospho-Smad3 antibody. Total Smad2/3 was used as a loading control. Band density was quantified and fold changes over 1-h hypoxia were calculated. (d) PC3 cells were pretreated with ALK4/5 inhibitor SB431542 for 30 min, followed by treatments with TGF- $\beta 1$ and hypoxia for $7 \mathrm{~h}$. VEGFA 165 in CM were determined by ELISA. Data were calculated as fold changes over the basal level of secreted VEGFA ${ }_{165}$. Data are expressed as mean \pm SEM (b: $n=6 ; \mathbf{d}: n=3$ ). ANOVA and Duncan's modified range tests were used. Significant differences between groups in a given category $(P<0.05)$ are designated with different lowercase letters. CM, conditioned media; ELISA, enzyme-linked immunoassay; PVDF, polyvinylidene fluoride; RT-PCR, reverse transcription PCR; SDS-PAGE, sodium dodecyl sulfate-polyacrylamide gel electrophoresis; TGF- $\beta 1$, transforming growth factor- $\beta 1$; VEGFA, vascular endothelial growth factor A.

Effects of exogenous VEGFA 165 on cell proliferation and migration were assessed. As shown in Figure 4a, VEGFA 165 induced a 1.8-fold increase in cell migration at a dose of $0.2 \mathrm{ng} \mathrm{ml}^{-1}$ when compared with the control group. The maximal response was observed when PC3 cells were treated with $1 \mathrm{ng} \mathrm{ml}^{-1}$ VEGFA $_{165}$. The effect of VEGFA ${ }_{165}$ on cell migration was completely blocked by pretreatment with $10 \mathrm{nmol}^{-1}$ Ki8751, a specific VEGFR-2 tyrosine kinase inhibitor (Figure 4b). VEGFA $_{165}$ had no effect on the proliferation of PC3 cells, as determined by ${ }^{3} \mathrm{H}$-thymidine incorporation and MTT assays (data not shown).

To investigate whether TGF- $\beta 1$ and hypoxia induce prostate cancer cell migration via endogenous VEGFA, CM were collected from PC3 cells treated with TGF- $\beta 1\left(1 \mathrm{ng} \mathrm{ml}^{-1}\right)$, hypoxia, or hypoxia plus TGF- $\beta 1$ for $7 \mathrm{~h}$. Soluble VEGFR-2 $\left(17 \mathrm{ng} \mathrm{ml}^{-1}\right)$ and Ki8751were used to block VEGFA signaling. ${ }^{22,23}$ Whereas TGF- $\beta 1$ - and hypoxia-treated conditioned media were shown to induce 1.5 -fold increases in migration of PC3 cells, the conditioned media from the cells treated with hypoxia plus TGF- $\beta 1$ increased migration of PC3 cells by two fold (Figure 4c). These effects of conditioned media were blocked by soluble VEGFR-2 and Ki8751.

\section{DISCUSSION}

Hypoxia, TGF- $\beta 1$ and VEGF are important factors of the tumor microenvironment that regulate cancer progression and metastasis. Hypoxia occurs concomitant with tumor growth. ${ }^{1}$ It is interesting to note that a hypoxic phenotype has been observed in the tumor specimens from prostate cancer patients with poor prognosis. ${ }^{24}$ TGF- $\beta 1$ is a growth factor that is overexpressed in prostate cancer. $^{25}$ The general function of TGF- $\beta 1$ is to inhibit cell proliferation and to induce apoptosis. However, when the malignant tissue progresses to the advanced stages it acquires the ability to escape the tumor-suppressing activities of TGF- $\beta 1$, and to convert TGF- $\beta 1$ into a tumorigenic growth factor. ${ }^{26}$ VEGF family members, including VEGFA and VEGFC, have been shown to play important roles in prostate cancer progression. ${ }^{14,27}$ VEGFC binds to VEGFR-3 (Flt4) on lymphatic endothelial cells, and the VEGFC/VEGFR-3 signaling is specific for lymphoangiogenesis. ${ }^{28}$ TGF- $\beta 1$ inhibits lymphagiogenesis. ${ }^{29}$ In addition, hypoxia does not regulate VEGFC mRNA expression. ${ }^{30}$ Therefore, the VEGFC/VEGFR-3-dependent pathway is unlikely associated with the tumorigenic effects of hypoxia and TGF- $\beta 1$. On the other hand, hypoxia and TGF- $\beta 1$ regulate VEGFA 
a

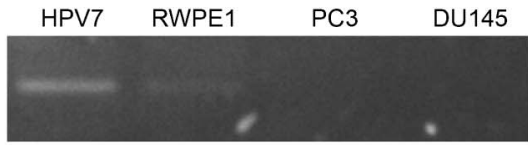

VEGFR2

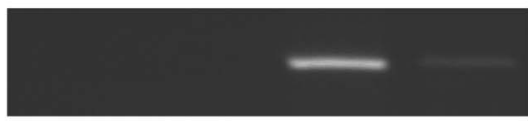

L19

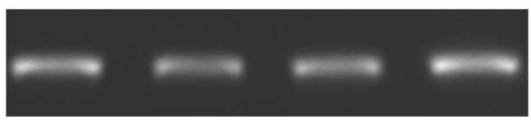

b

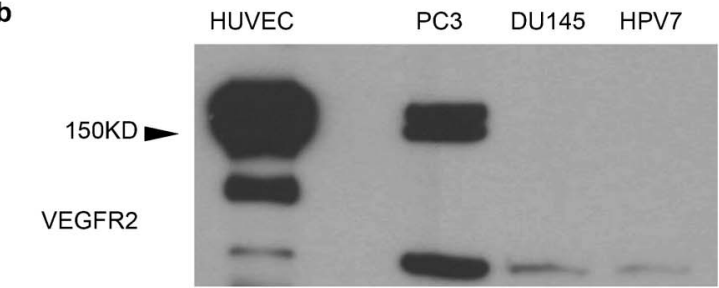

a-Tubulin

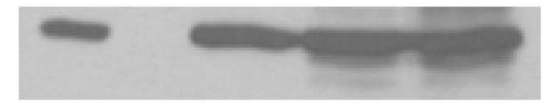

c
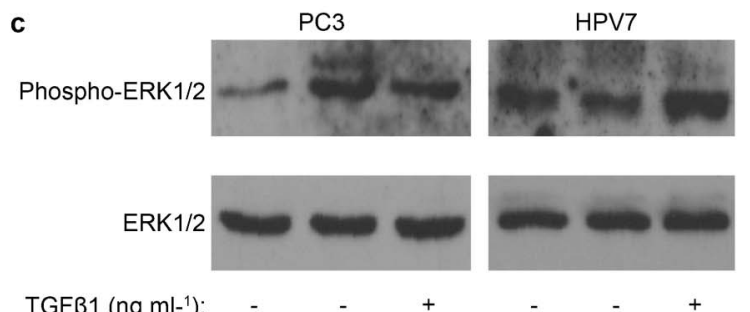

VEGF $\left(50 \mathrm{ng} \mathrm{ml}^{-1}\right)$ :

Folds:

\begin{abstract}
2.51
\end{abstract}
1.45

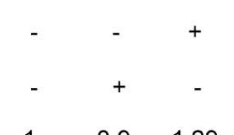

$\begin{array}{lll}1 & 0.9 & 1.29\end{array}$
Figure 3 Expression profiles of VEGFRs in prostate cell lines. (a) Levels of mRNA in HPV7, RWPE1, PC3 and DU145 cell lines were determined by RT-PCR. Similar results were replicated in three independent experiments. (b) Membrane protein fractions ( $\sim 60 \mu \mathrm{g}$ ) of HPV7, DU145 and PC3 cells and total cell proteins ( $\sim 30 \mu \mathrm{g}$ ) of HUVECs were used for western blot to detect expression of VEGFR-2 protein with anti-VEGFR2 antibody. Similar results were observed with two independent membrane preparations. (c) PC3 and HPV7 cell lines were treated with TGF- $\beta 1$ for 7 h or VEGFA 165 for 10 min. Phosphorylated and total ERK1/2 were detected in samples $(30 \mu \mathrm{l})$ with anti-phospho-ERK1/2 ( 1 : 2000) and anti-ERK1/2 ( $1: 5000)$ antibodies, respectively. Band density was quantified, and fold changes over control groups were calculated. Similar results were replicated in two independent experiments. ERK1/2, extracellular signal-regulated kinase 1/2; HUVEC, human umbilical vein endothelial cell; RT-PCR, reverse transcription PCR; TGF- $\beta 1$, transforming growth factor- $\beta 1$; VEGFA, vascular endothelial growth factor A; VEGFR, vascular endothelial growth factor receptor.

gene expression, ${ }^{7,8}$ which may account for the tumorigenic potential of both stimuli.

There is limited information about the effect of hypoxia and TGF- $\beta 1$ on VEGFA secretion in advanced prostate cancer. DU145 and PC3 prostate cancer cell lines are representative cell models of castration resistant prostate cancer. RWPE1 and HPV7 are immortalized prostate luminal epithelial cell lines. VEGFA 165 protein was detectable in both conditioned media and cell lysates from all cell lines examined. The majority of $\mathrm{VEGFA}_{165}$ was in the secreted form. TGF- $\beta 1$ induced VEGFA 165 secretion in both normal and prostate cancer cell lines; however, the effect of hypoxia was only observed in the prostate cancer cell lines. These data support that VEGFA contributes to the effect of hypoxia and TGF- $\beta 1$ in prostate cancer cells. In the prostate cancer cell lines, TGF- $\beta 1$ and hypoxia exerted an additive effect on VEGFA $_{165}$ secretion, which is presumably mediated by different intracellular mechanisms. ${ }^{7}$ Because hypoxia increases TGF- $\beta 1$ expression in osteoblast and hepatoma cells, ${ }^{10,11}$ it is also conceivable that TGF- $\beta 1$ signaling might constitute a positive feedback loop to reinforce the effect of hypoxia on VEGFA $_{165}$ secretion in prostate cancer. In addition, an autocrine effect of TGF- $\beta 1$ has been reported to increase VEGFA secretion in DU145 and PC3 cell lines during the preparation of this manuscript. ${ }^{20}$ In the present study, hypoxia increased levels of TGF- $\beta 1$ mRNA and protein in PC3 cells. The involvement of TGF- $\beta$ signaling in the effects of hypoxia was supported by the induction of Smad3 phosphorylation in PC3 cells. Furthermore, the ALK4/5 inhibitor (SB431542) partially inhibited the effect of hypoxia on VEGFA $_{165}$ secretion in PC3 cells. Therefore, an autocrine TGF- $\beta 1$ signaling mechanism appears to be involved in hypoxia-mediated VEGFA $_{165}$ secretion.

Although PC3 cells are a model for a more metastatic prostate cancer. ${ }^{18,31}$ DU145 was shown to secrete 10 times more VEGFA 165 than normal prostate epithelial cells, and PC3 cells produced the least amount of VEGFA 165 . In line with our observation, VEGFA 165 has previously been shown to be three times higher in DU145 than PC3M (a derivative of PC3) cells. ${ }^{31}$ Nevertheless, the PC3M-derived tumor bears more aggressive phenotype and higher angiogenesis capacity than the one originated from DU145 cells in mouse xenografts. ${ }^{31}$ Although VEGFA expression correlates with high Gleason grade prostate cancer specimens, ${ }^{32}$ increased expression of VEGFA alone is not sufficient to promote prostate cancer progression. Autocrine VEGFA signaling involving neuropilin-1 has been implicated in the process of epithelial to mesenchymal transition in PC3 cells. ${ }^{32}$ Neuropilin-1 has been shown to facilitate VEGFA signaling through interaction with VEGFR-2. ${ }^{2}$ In the current study, VEGFR-2 protein was detected in PC3 but not in DU145 cell lines. These data support a complementary role of VEGFR-2 to promote prostate cancer progression.

ERK1/2 phosphorylation is a common phenomenon following activation of receptor tyrosine kinases. $^{33}$ Treatment with VEGFA $_{165}$ induced ERK1/2 phosphorylation in PC3 cells but not in HPV7 cells. These data supports an acquisition of autocrine VEGFA signaling involving VEGFR-2 in PC3 prostate cancer cells. VEGFA induces cell proliferation in epidermal tumors, which has been shown to be mediated by VEGFR-1..$^{5}$ In the present study, VEGFA $_{165}$ did not affect the proliferation of PC3 cells. On the other hand, VEGFA 165 was shown to induce the migration of PC3 cells. This effect of VEGFA 165 was completely blocked by pretreatment with the VEGFR-2 kinase inhibitor (Ki8751). In addition, conditioned media collected from hypoxia- and TGF$\beta 1$-treated cells were shown to induce migration of PC3 cells, and these effects were completely blocked by soluble VEGFR-2 and Ki8751. Hence, VEGFA/VEGFR-2-dependent signaling may contribute to the tumorigenic effect of hypoxia and TGF- $\beta 1$ to promote the metastasis of prostate cancer cells. A similar VEGFA/VEGFR-2 autocrine loop has previously been implicated in migration of LNCaP cells and its metastatic derivative C4-2 cell line. $^{16}$

A working model was showed in Figure 5, hypoxia and TGF- $\beta 1$ increase VEGFA secretion in prostate cell lines. Hypoxia also increases TGF- $\beta 1$ expression in PC3 prostate cancer cells, which 


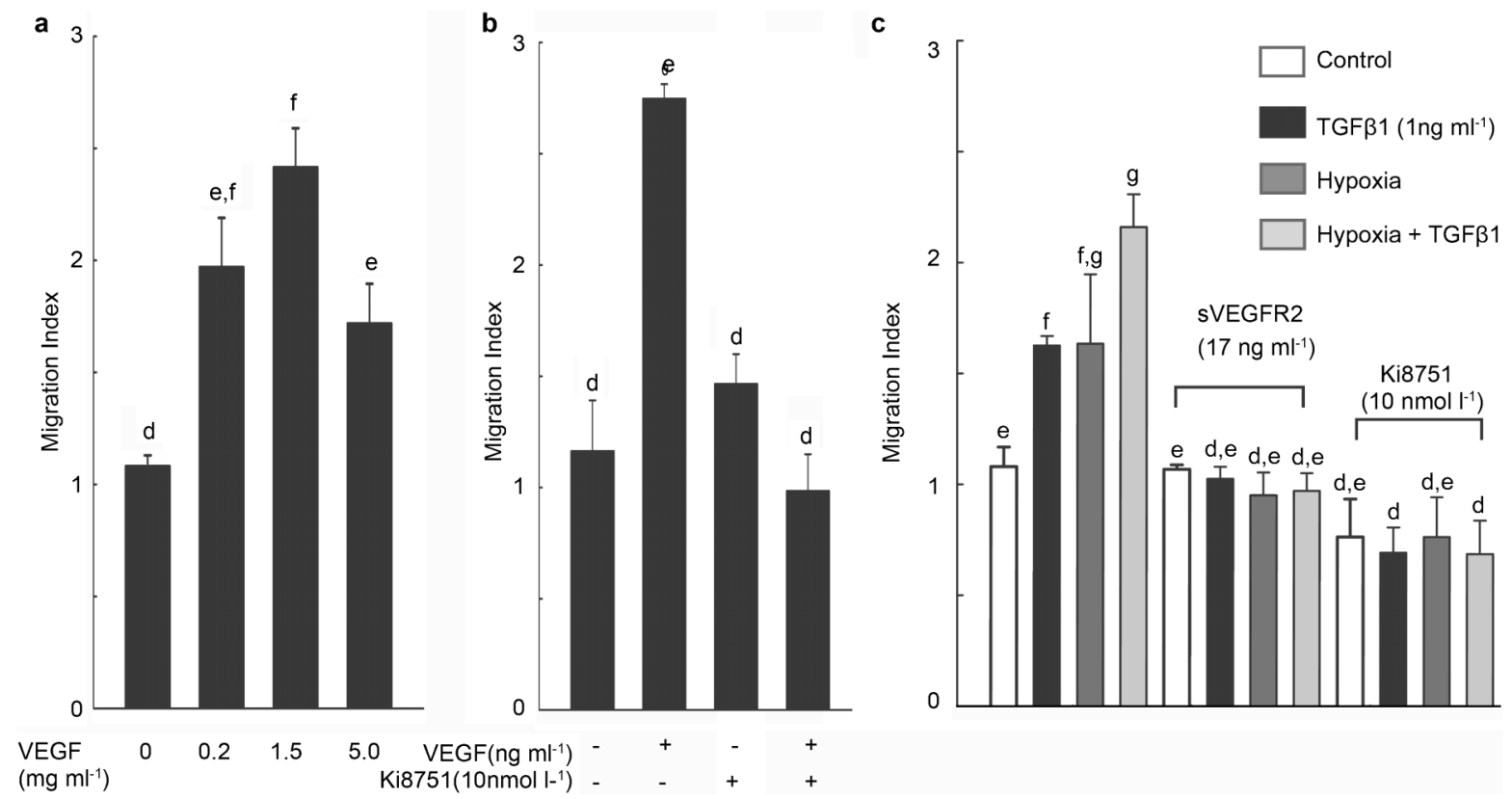

Figure 4 VEGFA induces PC3 cell migration via VEGFR-2 activation. PC3 cells $\left(3 \times 10^{5}\right.$ per transwell insert) were (a) treated with different concentrations of recombinant VEGFA 165 for $5 \mathrm{~h}$ or (b) pretreated with the VEGFR-2 kinase inhibitor Ki8751 for 30 min before treatment with VEGF 165 . (c) CM from TGF- $\beta 1$, hypoxia, or hypoxia plus TGF- $\beta 1$-treated PC3 cells were used to induce migration of PC3 cells in the absence/presence of soluble VEGFR-2 or Ki8751. Data are presented as mean \pm SEM ( $\mathbf{a}$ and $\mathbf{b}: n=4 ; \mathbf{c}: n=3$ ), and were analyzed by ANOVA and Duncan's modified range test. Significant differences between groups in a given category $(P<0.05)$ are designated with different lowercase letters. CM, conditioned media; TGF- $\beta 1$, transforming growth factor- $\beta 1$; VEGFA, vascular endothelial growth factor A; VEGFR, vascular endothelial growth factor receptor.

constitutes an autocrine mechanism to enhance the effects of hypoxia on VEGFA expression. This crosstalk between TGF- $\beta 1$ and hypoxia is novel and distinct from the established intracellular crosstalk to regulate VEGFA gene expression. ${ }^{7}$ The present study also suggests that VEGFA/VEGFR-2 autocrine loop partially accounts for the tumorigenic effects of TGF- $\beta 1$ and hypoxia in prostate cancer progression.

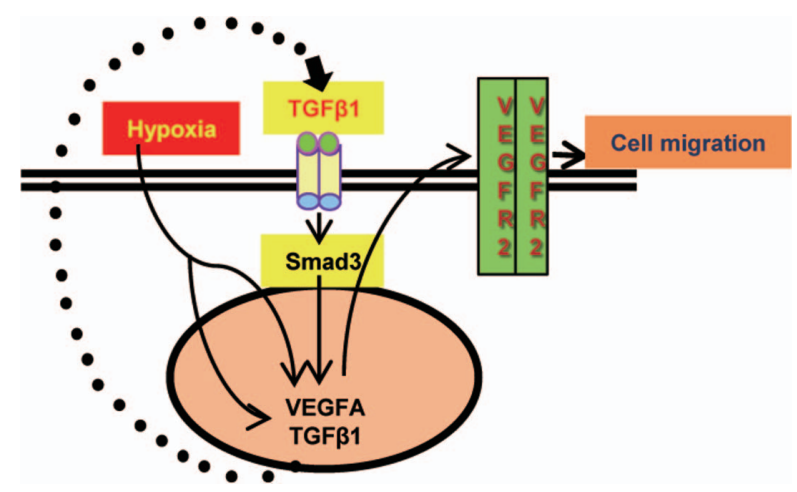

Figure 5 A diagram illustrating how hypoxia and TGF- $\beta 1$ become tumorigenic in advanced prostate cancer cells to increase tumor metastasis. PC3 prostate cancer cell line expresses VEGFR-2 protein that enables an autocrine VEGFA signaling in PC3 cells. The autocrine VEGFA/VEGFR-2 mechanism is important for cell migration in response to different stimuli, such as hypoxia and TGF- $\beta 1$. In addition to directly induce VEGFA expression, hypoxia triggers an autocrine TGF- $\beta 1$ signaling to increase VEGFA expression in prostate cancer cells. TGF- $\beta 1$, transforming growth factor- $\beta 1$; VEGFA, vascular endothelial growth factor A; VEGFR, vascular endothelial growth factor receptor.

\section{AUTHOR CONTRIBUTIONS}

BHV analyzed different treatments on Smad-3 phosphorylation (Figure $2 \mathrm{c}$ ), and detected $\alpha$-tubulin (Figure $3 \mathrm{~b}$ ). She also made the cartoon of Figure 5. ED and MZ performed the rest of experiments. $\mathrm{MZ}$ performed data statistical analysis. MZ and SAK wrote the manuscript. SK provided laboratory resources.

\section{COMPETING FINANCIAL INTERESTS}

There are no professional affiliations that would bias this presentation.

\section{ACKNOWLEDGMENTS}

This work is supported by NIH (RCMI 5G12RR003062 and NIMHD 1P20MD002285) and Georgia Research Alliance.

1 Ferrara N, Gerber HP, LeCouter J. The biology of VEGF and its receptors. Nat Med 2003; 9: 669-76.

2 Soker S, Takashima S, Miao HQ, Neufeld G, Klagsbrun M. Neuropilin-1 is expressed by endothelial and tumor cells as an isoform-specific receptor for vascular endothelial growth factor. Cell 1998; 92: 735-45.

3 de Vries C, Escobedo JA, Ueno H, Houck K, Ferrara $\mathrm{N}$ et al. The fms-like tyrosine kinase, a receptor for vascular endothelial growth factor. Science 1992; 255: 98991.

4 Terman BI, Dougher-Vermazen M, Carrion ME, Dimitrov D, Armellino DC et al. Identification of the KDR tyrosine kinase as a receptor for vascular endothelial cell growth factor. Biochem Biophys Res Commun 1992; 187: 1579-86.

5 Lichtenberger BM, Tan PK, Niederleithner H, Ferrara N, Petzelbauer P et al. Autocrine VEGF signaling synergizes with EGFR in tumor cells to promote epithelial cancer development. Cell 2010; 140: 268-79.

6 Maxwell PH, Dachs GU, Gleadle JM, Nicholls LG, Harris AL et al. Hypoxia-inducible factor-1 modulates gene expression in solid tumors and influences both angiogenesis and tumor growth. Proc Natl Acad Sci U S A 1997; 94: 8104-9.

7 Dunn LK, Mohammad KS, Fournier PG, McKenna CR, Davis HW et al. Hypoxia and TGF-beta drive breast cancer bone metastases through parallel signaling pathways in tumor cells and the bone microenvironment. PLoS One 2009; 4: e6896. 
8 Sanchez-Elsner T, Botella LM, Velasco B, Corbi A, Attisano L et al. Synergistic cooperation between hypoxia and transforming growth factor-beta pathways on human vascular endothelial growth factor gene expression. J Biol Chem 2001; 276 38527-35.

9 Cunha SI, Pardali E, Thorikay M, Anderberg C, Hawinkels L et al. Genetic and pharmacological targeting of activin receptor-like kinase 1 impairs tumor growth and angiogenesis. J Exp Med 2010; 207: 85-100.

10 Patel B, Khaliq A, Jarvis-Evans J, McLeod D, Mackness M et al. Oxygen regulation of TGF-beta 1 mRNA in human hepatoma (Hep G2) cells. Biochem Mol Biol Int 1994; 34: 639-44.

11 Warren SM, Steinbrech DS, Mehrara BJ, Saadeh PB, Greenwald JA et al. Hypoxia regulates osteoblast gene expression. J Surg Res 2001; 99: 147-55.

12 Duque JL, Loughlin KR, Adam RM, Kantoff PW, Zurakowski D et al. Plasma levels of vascular endothelial growth factor are increased in patients with metastatic prostate cancer. Urology 1999; 54: 523-7.

13 Pallares J, Rojo F, Iriarte J, Morote J, Armadans LI et al. Study of microvessel density and the expression of the angiogenic factors VEGF, bFGF and the receptors FIt- 1 and FLK-1 in benign, premalignant and malignant prostate tissues. Histol Histopathol 2006; 21: 857-65.

14 Sweeney P, Karashima T, Kim SJ, Kedar D, Mian B et al. Anti-vascular endothelia growth factor receptor 2 antibody reduces tumorigenicity and metastasis in orthotopic prostate cancer xenografts via induction of endothelial cell apoptosis and reduction of endothelial cell matrix metalloproteinase type 9 production. Clin Cancer Res 2002; 8: 2714-24.

15 Soker S, Kaefer M, Johnson M, Klagsbrun M, Atala A et al. Vascular endothelial growth factor-mediated autocrine stimulation of prostate tumor cells coincides with progression to a malignant phenotype. Am J Pathol 2001; 159: 651-9.

16 De S, Chen J, Narizhneva NV, Heston W, Brainard J et al. Molecular pathway for cancer metastasis to bone. J Biol Chem 2003; 278: 39044-50.

17 Zhang S, Zhau HE, Osunkoya AO, Iqbal S, Yang X et al. Vascular endothelial growth factor regulates myeloid cell leukemia-1 expression through neuropilin-1-dependent activation of c-MET signaling in human prostate cancer cells. Mol Cancer 2010; 9: 9

18 Zhong M, Boseman ML, Millena AC, Khan SA. Oxytocin induces the migration of prostate cancer cells: involvement of the Gi-coupled signaling pathway. Mol Cancer Res 2010; 8: 1164-72.

19 Zhong M, Navratil AM, Clay C, Sanborn BM. Residues in the hydrophilic face of putative helix 8 of oxytocin receptor are important for receptor function. Biochemistry 2004; 43: 3490-8.

20 Chae KS, Kang MJ, Lee JH, Ryu BK, Lee MG et al. Opposite functions of HIF-alpha isoforms in VEGF induction by TGF-beta1 under non-hypoxic conditions. Oncogene 2011; 30: 1213-28.
21 Laping NJ, Grygielko E, Mathur A, Butter S, Bomberger J et al. Inhibition of transforming growth factor (TGF)-beta1-induced extracellular matrix with a novel inhibitor of the TGF-beta type I receptor kinase activity: SB-431542. Mol Pharmacol 2002; 62: 58-64.

22 Aiello LP, Pierce EA, Foley ED, Takagi $\mathrm{H}$, Chen $\mathrm{H}$ et al. Suppression of retinal neovascularization in vivo by inhibition of vascular endothelial growth factor (VEGF) using soluble VEGF-receptor chimeric proteins. Proc Natl Acad Sci U S A 1995; 92 10457-61.

23 Kubo K, Shimizu T, Ohyama S, Murooka H, Iwai A et al. Novel potent orally active selective VEGFR-2 tyrosine kinase inhibitors: synthesis, structure-activity relationships, and antitumor activities of $N$-phenyl- $N$ '-\{4-(4-quinolyloxy)phenyl\}ureas. J Med Chem 2005; 48: 1359-66.

24 Nanni S, Benvenuti V, Grasselli A, Priolo C, Aiello A et al. Endothelial NOS estrogen receptor beta, and HIFs cooperate in the activation of a prognostic transcriptional pattern in aggressive human prostate cancer. J Clin Invest 2009; 119: 1093-108.

25 Eastham JA, Truong LD, Rogers E, Kattan M, Flanders KC et al. Transforming growth factor-beta 1: comparative immunohistochemical localization in human primary and metastatic prostate cancer. Lab Invest 1995; 73: 628-35.

26 Pardali K, Moustakas A. Actions of TGF-beta as tumor suppressor and pro-metastatic factor in human cancer. Biochim Biophys Acta 2007; 1775: 21-62.

27 Tuomela J, Valta M, Seppanen J, Tarkkonen K, Vaananen HK et al. Overexpression of vascular endothelial growth factor $\mathrm{C}$ increases growth and alters the metastatic pattern of orthotopic PC-3 prostate tumors. BMC Cancer 2009; 9: 362.

28 Wong SY, Haack H, Crowley D, Barry M, Bronson RT et al. Tumor-secreted vascular endothelial growth factor- $C$ is necessary for prostate cancer lymphangiogenesis, but lymphangiogenesis is unnecessary for lymph node metastasis. Cancer Res 2005; 65: 9789-98.

29 Clavin NW, Avraham T, Fernandez J, Daluvoy SV, Soares MA et al. TGF-beta1 is a negative regulator of lymphatic regeneration during wound repair. Am J Physiol Heart Circ Physiol 2008; 295: H2113-27.

30 Enholm B, Paavonen K, Ristimaki A, Kumar V, Gunji Y et al. Comparison of VEGF, VEGF-B, VEGF-C and Ang-1 mRNA regulation by serum, growth factors, oncoproteins and hypoxia. Oncogene 1997; 14: 2475-83.

31 Connolly JM, Rose DP. Angiogenesis in two human prostate cancer cell lines with differing metastatic potential when growing as solid tumors in nude mice. J Urol 1998; 160: 932-6.

32 Mak P, Leav I, Pursell B, Bae D, Yang X et al. ERbeta impedes prostate cancer EMT by destabilizing HIF-1alpha and inhibiting VEGF-mediated snail nuclear localization: implications for Gleason grading. Cancer Cell 2010; 17: 319-32.

33 Hurlbut GD, Kankel MW, Artavanis-Tsakonas S. Nodal points and complexity of NotchRas signal integration. Proc Natl Acad Sci U S A 2009; 106: 2218-23. 